\title{
How Cryptocurrencies Are Changing What CPAs Need to Know about Fraud Prevention
}

\author{
Sean Stein Smith \\ Department of Economics and Business, Lehman College, City University of New York, New York, NY, USA \\ Email: ssteinsmith@y ahoo.com
}

How to cite this paper: Smith, S.S. (2018) How Cryptocurrencies Are Changing What CPAs Need to Know about Fraud Prevention. Theoretical Economics Letters, 8, 3252-3266.

https://doi.org/10.4236/tel.2018.814201

Received: August 22, 2018

Accepted: October 28, 2018

Published: October 31, 2018

Copyright $\odot 2018$ by author and Scientific Research Publishing Inc. This work is licensed under the Creative Commons Attribution International License (CC BY 4.0).

http://creativecommons.org/licenses/by/4.0/

\begin{abstract}
The accounting profession has long occupied a role and fiduciary responsibility aligned with fraud prevention and safeguarding consumer data and finances. As cryptocurrencies become more prominent and widespread, including utilization by both individual consumers and organizations, the risk of unethical actors entering the market continues to rise. This research approaches the situation from two angles. First, a review of cryptocurrency and blockchain technology, including an overview of the initial coin offering (ICO) process is conducted, including regulatory updates in the space. Second, and important for both academic and practitioner end users of this research, a checklist, guide, and items to consider to assist in preventing fraud connected to cryptocurrencies will be provided.
\end{abstract}

\section{Keywords}

Cryptocurrencies, Accounting, Fraud, Ethics, Financial Reporting

\section{Introduction}

Cryptocurrencies may have seized the attention of the mass media and market in 2016 and 2017 but the concept of cryptocurrencies is not, in and of itself, an entirely new idea or concept. Linking together the idea of a peer-to-peer payment and communication system that can occur and exist without the need for a central clearing house is, potentially, a revolutionary idea in the context of financial services. The vast majority of work performed and done by accounting, finance, and banking professionals focuses on the validation, confirmation, and communication of large amounts of quantitative information. In addition to the core functionalities of encryption, the ability to remove a central clearing house from the conversation, and the anonymity that is granted via cryptocurrencies, there must also be a discussion and analysis of how cryptocurrencies can also lead to 
unethical and fraudulent behavior.

On top of the implications of cryptocurrencies generating opportunities for fraud due to the lack of regulation and oversight, it is also important to recognize the reality that, as the overall market and scope of cryptocurrencies continues to increase and mature, additional applications continue to be built on the foundation of cryptocurrencies. Specifically, the process of initial coin offerings (ICOs, in which organizations raise money from the capital market in exchange for tokens or other type of cryptocurrencies has already created an environment where unethical behavior is underway. This does not appear to be merely an academic or theoretical discussion due to the sheer volume of cryptocurrencies that have already been issued and introduced to the marketplace. With over 900 cryptocurrencies launched in 2017, and over 50\% of those cryptocurrencies having failed by mid-2018, there are multiple instances in which investors have been defrauded.

This research drills into the core functionality of cryptocurrencies, including how different applications and next step uses related to cryptocurrency can actually contribute to unethical, fraudulent, and other unwanted business activity. Additionally, and after an analysis and examination of current literature in the areas of cryptocurrencies and potential fraudulent behavior, accounting specific applications will also be covered. While blockchain and cryptocurrencies are already having an impact on the accounting and finance, the specific implications as they connect to unethical and fraudulent behavior remain an emerging area. Following a review and analysis of literature and market based evidence linked to cryptocurrency applications, guidelines and suggestions will also be presented in order to assist practitioners seeking to address possible gaps and unethical actions by market participants.

\section{Literature Review}

Although the field of cryptocurrencies and cryptocurrency applications are still an emerging area of both study and practitioner research, it is appropriate to conduct a thorough review of current research and findings. Cryptocurrencies may and the underlying blockchain technology may be computer science based and focused in nature, but there has been quite a bit of discussion, research, and debate among accounting and finance professionals. Drilling down specifically, it appears logical to construct a literature review focusing not only on what cryptocurrencies are, but also the use cases and applications for financial services.

\section{Blockchain}

Regardless of what industry is examined, or the specific organizations that comprise the majority of that analysis, it appears increasingly evident that blockchain technology may represent the largest paradigm shift in financial services since the implementation of computing for accounting firms and practitioners [1]. An analysis of blockchain technology can, and often is, the focus of entire academic and practitioner articles, and while an exhaustive analysis of 
blockchain is not the purpose of this research, an initial discussion and overview appears appropriate. At this point, however, and in spite of the rapid increase in investment and interest in the blockchain space there are still obstacles that may hamper innovation and adaption of the technology. The core functionality of blockchain technology revolves around several fundamental traits and characteristics that differentiate this technology tool from current offerings. Drilling down, these traits and characteristics include, but are not limited to the following:

1) Decentralized-an underlying force linked to blockchain technology, and one that is radically different than any other existing options in the marketplace is that blockchain technology itself is, at least conceptually, a totally decentralized concept. Even in the context of a private blockchain, with an organizing firm writing guidelines and inviting members, a large degree of the actions undertaken are decentralized in nature. Stated another way, there is not one central hub or regulatory authority in charge of resolving issues, complaints, unethical activity and other operational issues.

2) Consensus based verification-in addition to the decentralized nature of blockchain technology, in and of itself differentiated from other market options, information added to a blockchain must also be verified by other members of the network. The specifics can, and often do, vary from blockchain to blockchain, but regardless of the specific protocol in place, underlying facts are similar. Boiling it down to the key action points, in order for data to be added by any one individual member of the blockchain network, other members must mathematically verify that the information is both accurate and connected (via hash ID) to previous blocks. Specific approval protocols can, and do, vary from blockchain to blockchain, but consensus is an important concept regardless of protocol specifics.

3) Encryption-perhaps the most obvious connection and benefit of blockchain technology are the encryption protocols that both secure information and enable the system of centralized clearinghouses to be replaced by a peer-to-peer network system. The encryption protocols that are a part of blockchain technology represent both an opportunity and a challenge to financial services professionals. The appeal of a decentralized model is clear to consumers but can also create gaps and opportunities that accounting professionals should be aware of going forward. Drilling down, due to the anonymous nature of cryptocurrency itself, unethical behavior and fraudulent actions may not be noticed until significant damage has been incurred.

4) Basis for new technologies - despite all of the analysis and examination of blockchain technology already underway in the marketplace, it is important to note the reality that many industries and applications are still in early stages of actual implementation and application. Building off of the fact, this research drills down into exactly how some of the future oriented applications, including but not limited to ICOs can, in addition to building on blockchain, also create opportunities for unethical behavior.

It also appears logical to illustrate and highlight at this point that, although 
blockchain technology may seem to represent an entirely new technology, it also represents a combination of existing aspects of technology in the market. That said, blockchain does represen a differentiated technology tool and platform that is different from existing tools as outlined in Table 1. An approach and mindset that seems to be quite common in the marketplace is a connection between big data, data analytics, and the approaches that accounting organizations will need to take to combine these technological forces [2]. One iteration that may represent true innovation in the space of blockchain, cryptocurrency, and implications for accounting professionals is the concept of an initial coin offering (ICO). In addition to representing market innovation and additional options for entrepreneurs seeking to raise capital to fund and maintain a business, the process of an ICO also represents an opportunity for possible fraud and unethical behavior. To better understand the linkages between these different areas, and the implications ICOs and cryptocurrencies may have on accounting and legal services professionals, it seems appropriate to drill down into what exactly an ICO represents.

\subsection{Initial Coin Offerings and Cryptocurrencies}

While it is true that blockchain technology has been around in the marketplace since the mid-2000s, perhaps the most high profile and scalable iteration of blockchain technology has been the cryptocurrency market. The overall value of the cryptocurrency market continues to fluctuate due to the volatility of individual assets, but it does appear evident that the asset class at large continues to increase [3]. Whether it takes the form of Bitcoin, Ether, XRP, or some other form version of cryptocurrencies the underlying components are well known by market participants at this stage. Trading differences in different markets, regulatory uncertainty, a variety of accounting options (both for financial and tax reporting purposes), and the different legal frameworks used in different nations have created a web of uncertainty that could, and does, form the basis for numerous articles. This specific research, however, focuses on the growing interest in ICOs, applications for cryptocurrencies, and how the ICO process itself can generate implications for accounting professionals from both an accounting and legal perspective.

Table 1. Characteristics of blockchain versus traditional technology tools.

\begin{tabular}{|c|c|c|}
\hline Characteristic & Differences from current technology & Applications for CPAs \\
\hline Decentralized & $\begin{array}{l}\text { Yes, fundamentally different from centralized technology, } \\
\text { financial, and data management systems }\end{array}$ & $\begin{array}{l}\text { No central oversight or resolution authority for disputes or } \\
\text { possible fraud activity }\end{array}$ \\
\hline Consensus & $\begin{array}{l}\text { No central approver or verifier of information, instead } \\
\text { network members verify data as it is uploaded }\end{array}$ & $\begin{array}{l}\text { As data is uploaded, members of the network it is verified } \\
\text { continuously by other network members }\end{array}$ \\
\hline Encrypted & $\begin{array}{l}\text { The encryption is a integral component of blockchain } \\
\text { technology, specifically the } 256 \text { bit protocol of the } \\
\text { Bitcoin blockchain }\end{array}$ & $\begin{array}{l}\text { To date, the encryption associated with the blockchain has } \\
\text { yet to be hacked }\end{array}$ \\
\hline New technologies & $\begin{array}{l}\text { Flexibility of blockchain allows for different applications and } \\
\text { iterations to leverage this technology }\end{array}$ & $\begin{array}{l}\text { Traditional technology tools operate in siloed environment, } \\
\text { and are not compatible with other platforms }\end{array}$ \\
\hline
\end{tabular}


ICOs, representing a relatively new application and build out based on blockchain technology, and integrating cryptocurrencies within the financial markets, is an area ripe with both misunderstanding and the potential for unethical activity. Before diving into some of the operational details of how an ICO process works, it seems important to also state the scale of the size of this marketplace. Although many of the cryptocurrencies that have launched during the cryptocurrency boom of 2017 and 2018 have failed, flopped, and led to investor losses, the market continues to grow [4], interest in the space continues to increase. With the number of ICOs already have been launched with a total market in the hundreds of billions, this does not appear to be merely an academic or theoretical discussion. Some key points to assist with understanding some of the mechanics of the ICO process are as follows:

First, ICOs are not the same as an IPO-although the concept of an IPO is one that virtually every practitioner involved in the financial services landscape, the idea of an ICO is something that still may remain murky. In a traditional IPO the organization seeking to raise capital exchanges ownership in the firm for cash provided by investors, and investors than can share in the future profits of the organization. An ICO, however, although similar in labeling and basic function-the exchange of financial assets for an organizational asset-does have significant differences. A core difference and a difference that has to date driven the majority of regulatory discussion to date are the concept of a token. This second point, the issuance and exchange of a token for cash and financial assets, is perhaps the most high profile difference between a coin offering and a public offering.

A token, boiling down to the core terms and idea of what exactly it means from a business perspective, can be thought of as an option. A token is, in essence, an option that gives the investors in the organization the right to something at a date in the future; products, services, or ownership in the firm at some date in the future all are options that can be aligned with the issuance of a token. This new asset class, alongside the rise and popularity of cryptocurrencies, has also led to increased scrutiny and regulation around the issuance and trading of tokens, especially the implementation of a new asset class labeled as utility tokens. Table 2 breaks down some of the key questions and items CPAs and other accounting practitioners should raise when discussing both the concepts themselves as well as how ICOs are different from IPOs. This conversation and debate, around just how to classify and regulate different classes and types of crypto currencies and cryptoassets, continues to develop and evolve, so being aware of these changes is a responsibility for every fiduciarily responsible financial professional.

\subsection{Regulatory Landscape}

The regulatory landscape surrounding cryptocurrencies continues to evolve and shift in the face of both changing regulatory forces and the business use cases for cryptocurrencies and other blockchain applications [5]. Just one example 
Table 2. Key questions differentiating ICOs from IPOs.

\begin{tabular}{ccc}
\hline Key Questions & Initial Coin Offering & Initial Public Offering \\
\hline What is exchanged & $\begin{array}{c}\text { Cash for either 1) a cryptocurrency or 2) } \\
\text { a token issued by the organization }\end{array}$ & $\begin{array}{c}\text { Cash for some sort of equity ownership } \\
\text { in the organization }\end{array}$ \\
$\begin{array}{c}\text { Is there regulatory certainty for accounting } \\
\text { and reporting this transaction? }\end{array}$ & The regulatory environment is still evolving \\
Are there tax implications of this exchange? & Yes & The regulatory environment is certain \\
\hline
\end{tabular}

focused in the United States has to do with how, exactly, cryptocurrencies will be regulated by the Securities and Exchange Commission, just one of the major regulatory agencies in the U.S. In June of 2018 the SEC issued a statement that was intended to clarify some of the uncertainty and murkiness surrounding the issue of cryptocurrency trading and investment. Put simply, the Commissions stated that cryptocurrencies that had been issued previously, and were truly decentralized and distributed in terms of ownership would not be treaded as securities. Rather, these decentralized cryptocurrencies would instead be treated as commodities, meaning that the oversight and regulation would not as be as onerous.

Interestingly, while the SEC did issue guidance that would reduce regulations on some existing cryptocurrencies, especially those that had been issues and outstanding for the longest (like Bitcoin), the other aspect of this guidance was on the same wavelength. For newer cryptocurrencies, including those formally issued via an ICO process, the regulation for those items would indeed mirror, to at least some extent, those of equity securities. This statement only added to the uncertainty around the regulation and operation of cryptocurrency oriented businesses, especially for new organizations seeking to increase the overall market for cryptoassets. Regulation continues to evolve and change, and on top of being differentiated on a country by country basis, remains murky with regards to just how these items should be treated for reporting and taxation purposes [6]. In addition to this bifurcation of the marketplace, it is also interesting to note that some regulation applies to newly issued cryptocurrencies that are issued with the desire to generate a profit. While it is clear that virtually every organization is motivated to either generate a profit, or at least produce more resources than it consumes, this delineation has also led to the proposing of a new class of tokens; utility tokens.

Utility tokens are, according to existing literature and research, including commentary from market actors, often propositioned as an alternative to issuing traditional security tokens. As current understanding has been clarified through market news and actors, utility tokens initially do not provide any value or utility to the marketplace, but are merely used as a placeholder until the organization can issue security tokens that are directly linked to the business itself. In other words, utility tokens are a tool to ensure that, as the organization seeks to raise capital and fund operations, that liquidity can be maintained as the organization expands, develops, and continues to seek funding. As per the most recent SEC 
iterations and guidance, however, there does not appear to be special class or regulation associated with utility tokens. At best, the issuance of a utility token can often muddy the water between tax authorities, regulators, and the management of the organization in question.

\subsection{Tax Reporting and Regulation}

Despite the variety of factors, forces, and trends that are in the marketplace, and potentially adding to the confusion and misunderstanding of the cryptocurrency marketplace is tax regulation. This research focuses on U.S. regulation, but it is also worth nothing that cryptocurrencies and ICOs are currently taxed and regulated in different ways depending on the national marketplace in question. This is of particular importance due to the global nature of blockchain and cryptocurrency technology itself; one of the core concepts connected to the idea of cryptocurrencies is to facilitate transactions and transmission of data across borders and between organizations. Circling back to the United States, however, does illustrate the apparent contraction between what cryptocurrencies are labeled as and how they are treated for tax reporting. Despite the labeling and usage of various cryptocurrencies by individuals for purchasing and selling different goods and services, under current I.R.S. regulations and guidelines all cryptocurrencies are currently taxed as property [7]. To a nonfinancial expert this may seem like a minor distinction, the difference between tax something as currency versus something as property, but the ramifications are profound. Without diving too much into the technical specifications, the main difference is that every time a cryptocurrency is transferred, sold, bought, or used in any capacity it may generate an individual taxable event. Although the taxation issues linked to cryptocurrency are not the focus of this research, it is logical to include them for the purposes of connecting the cryptocurrency trend, fraud implications, and role of accounting professionals in this changing landscape.

\section{Accounting Specific Implications}

Demonstrated throughout this research piece and analysis, the changing regulatory landscape offers both opportunities and challenge for management professionals, it also presents accounting practitioners with a variety of issues. A core responsibility of all accounting and finance professionals is to assist with the fiduciary management of assets and organizations; this much is readily apparent. An additional duty, however, is to also prevent fraud and other unethical activity from occurring within an organization, regardless of industry affiliation. Internal controls, policies and procedures to help protect assets, information, and other organizational information have been well developed over decades. With the rise and advent of the cryptocurrency landscape and broader crypto linked economy, however, the role and responsibility of accounting professionals will inevitably have to evolve and change over time. Specifically, accounting professionals and practitioners will need to engage in several different tactics to remain 
relevant in a business environment changing on a nearly continuous basis.

First, accounting professionals will need to become familiarized with the implications that the various iterations of cryptocurrency will have on financial reporting, accounting, and financial analysis. Real time transmission of information, the encryption of data, and the capability of individuals to transact with individuals anywhere in the world will obviously create clients with questions that accounting practitioners must be able to address. Tax implications, how to report different types of cryptocurrency related information, and the implications of cryptocurrency on how financial statements appear to external users are just some of the higher profile areas that cryptoassets will have an impact on the accounting profession. Second, introducing the second major ramifications of cryptocurrencies are the legal implications that will inevitably arise from the proliferation of cryptoassets, cryptocurrencies, and the broader implications of crypto on major economic trends at large.

Prior to diving directly legal implications, specifically those linked to fraud prevention and detection, it does appear logical to acknowledge the role accounting and accounting information systems will play in this process. Even though the underlying force driving cryptocurrency adoption and implementation does tend to be technological in nature, the financial responsibilities of users continue to expand. Namely, and to be expanded upon in this research, accounting professionals must be aware of what questions to ask, which factors to consider, and how to deal with issues as they arise in the course of conducting business [8]. Such a strategic headset is not unique to cryptocurrency issues, however, and instead point to a broader shift toward accounting and financial services professionals embracing a more strategic and partner focused role within different industries and organizations. Advising management professionals with insights and advice on implications of different strategic and tactics represent trends already present in the marketplace; these will merely be amplified by the increasing integration of technology throughout the business process. In order to offer comprehensive advice and guidance however, it is important that accounting professionals understand both the financial and legal impact cryptocurrencies will, and already are, having on client requirements.

\section{Legal Implications}

While accounting or financial services professionals do not regularly take positions on broad legal issues of interpretations or different statues, it is increasingly expected that said professionals can, and will, offer guidance on a changing regulatory landscape. Several legal implications of particular importance to the accounting profession include, but are not limited to the passage of the General Data Protection Regulation (GDPR), tax reform in the United States, and the increasing focus of regulators around the area of cryptocurrencies in general. Fraud and unethical behavior can always occur, and does occur with regularity regardless of the specific tools or platforms used, but the possibility of these be- 
haviors as they are connected to cryptocurrencies appears to only be growing in importance.In fact, there is evidence of certain cryptocurrency exchanges and organizations choosing to not operate or establish bases on operation in the United States due to the uncertainty associated with the current regulatory landscape [5]. Since the landscape itself, including the operation of exchanges as well as the regulatory backdrop of these businesses is still emerging itself, establishing a baseline from which other decisions could be made is imperative [5]. Drilling down specifically, the impact of potential fraudulent and unethical behavior is now expanding as institutional interest increases in the cryptocurrency space.

A working definition of fraud is important, especially as accounting practitioners seek to become more proactive and forward looking in the cryptocurrency space. For the purposes of this research, fraud and unethical behavior can be thought of as deliberate actions undertaken by market participants to deceive or otherwise take advantage other participants. While confronting and combating fraud has formed the basis for numerous technology systems and tools, doing so with regards to cryptocurrency remains an uncertain task. Put simply, the increased utilization of cryptocurrency and other cryptoassets means that the risk associated with virtual currency and these other assets is a business risk that must be managed. Legally speaking, one piece of information that needs to be considered is that different types of cryptocurrencies may very well be treated different under current legislation as mentioned previously. Accountants and/or fraud examiners will not be expected to offer legal advice in every situation, but having a working knowledge of how different regulation will impact the value of cryptocurrencies increasingly appears to be a working requirement of professionals.

\section{Preventing Unethical Behavior Connected to Cryptocurrencies}

Returning to the crux of this research, the creation of a checklist and guide for accounting practitioners seeking to prevent fraud or unethical activities connected with cryptocurrencies, the following steps appear logical. Something that should be of particular importance and noted by practitioners is that, a strength of cryptocurrency, the disintermediation of traditional clearing houses, may also create a situation in consumers and organizations do not have a central hub or clearing house to help them resolve any potential issues [9]. This listing of suggestions and activities is not meant to be all inclusive nor exhaustive, but rather should form the basis for a comprehensive dialogue about accounting regulations, cryptocurrencies, and maintaining the fiduciary role of accountants in preventing the misuse of frauds. As smart contracts and other automated executable applications are constructed on top of a blockchain platform, the potential for blockchain as a fraud prevention tool, although such applications remain, for the time, as works in progress [10]. Drilling in specifically, it should 
include but is not limited to the following:

1) Understand why clients are investing in cryptoassets-there are specific use cases that have been built out around blockchain technology, and cryptocurrencies may be the most high profile example to date. That said, having an in-depth conversation with clients about the underlying purpose of investing in cryptocurrencies can, by itself, prevent some instances of fraudulent activities. Reviewing what may be considered the basics may seem like an initial seem redundant but may also uncover potential issues with plans designed to use cryptocurrencies before they cause financial harm.

2) Perform due diligence on any ICO activity - raising capital on a blockchain platform and doing so via cryptocurrencies or tokens has attracted large amounts of attention and investment, but due diligence is still necessary. Researching, not only the founders of the organization but other potential investors in the organizations are critically important for not only preserving capital but reducing any unexpected or unnecessary liability due to other unethical members partaking in the ICO process. Conversely, performing research and due diligence might be perceived as even more important when blockchain technology is involved, especially since the identities of other members may not be readily accessible as time moves forward.

3) Understand the trade between capital and what is received-based on the differences between the variety of cryptoassets that can be issued as part of an ICO, and the range of tax treatments possible as a result of these differences, ensuring clients understand what specifically is being received is critical. Linking back to the traditional role of accounting professionals as tax, reporting, and compliance experts, ensuring that information and data are communicated clearly between different participants will remain a core responsibility of accounting professionals.

4) Engage in continuing education and learning-from both a client perspective and a practitioner point of view embracing the field of blockchain and cryptocurrencies will be important but continuing to learn and professionally develop will arguably be more critical. Remaining up to speed on emerging issues and technology is, of course, well advised from a professional perspective, but is also necessary to provide timely and accurate guidance for clients.

5) Be aware of the crypto environment-cryptocurrencies may be the primary focus for individuals and even some organizations as far as blockchain technology is concerned, but for accounting practitioners being aware of the broader crypto business landscape is imperative. Blockchain and/or cryptocurrencies do not operate in a vacuum, nor are they excluded from business forces driving change and development in other areas. Akin to how different versions of operating software and accounting software emerge as market leaders over time, only to be supplanted by different versions later, a similar trend will inevitably occur within the crypto marketplace.

6) Keep on top of regulatory changes-as of this research and analysis the regulatory landscape continues to change both in terms of how cryptocurrencies 
are treated for tax purposes and financial reporting. Whether clients are simply investing in cryptocurrencies for speculative purposes, investing in ICOs as a part of the overall investment strategy, or are seeking to accept payments in cryptocurrencies the possibility of fraudulent activity will exist. CPAs and other accounting professionals must remain current and in position to offer up-to-date guidance.

Put simply, in order to provide clients, both current and former, with the insights required in a business environment increasingly dominated by cryptocurrencies, blockchain protocols, and other technology tools, accounting practitioners will need to evolve and keep pace alongside organizations. These lists of factors and items to consider, illustrated and cited in Figure 1, however, are not the only factors and items accounting professionals will need to consider. Rather, and particularly important for the conversation around potential fraudulent and unethical activity, applications relevant to business decision making at large need to also be analyzed.

\section{Business impact of the cryptoeconomy}

Unethical actions are not limited, however, to the mere exchange of cryptocurrencies for financial assets. In every situation where there is a motive for profit or an opportunity for some participants to engage in behavior to unduly benefit them there will be instances of fraud and unethical behavior. Taking a possible option, increased regulation, one step further the entire ICO process has been banned in China as a result of potential and actual fraudulent behavior associated with both individual cryptocurrencies and ICOs [6]. Accounting professionals, already playing a more strategic role in how business decisions are made across industry lines, should also be able to understand and advise on strategic decisions connected to cryptocurrencies. Circling back to a core pillar of this analysis is that while cryptocurrencies are a high profile example, the underlying blockchain technology is perhaps where the most potential and opportunity lies. While some of the implications have already been felt across different industry lines, broader effects still remain for the most parts work in progress.

For example, one of the core concepts of blockchain is that larger amounts of information will be more readily available than at previous times in business history. Financial data may for the basis for how financial professionals first
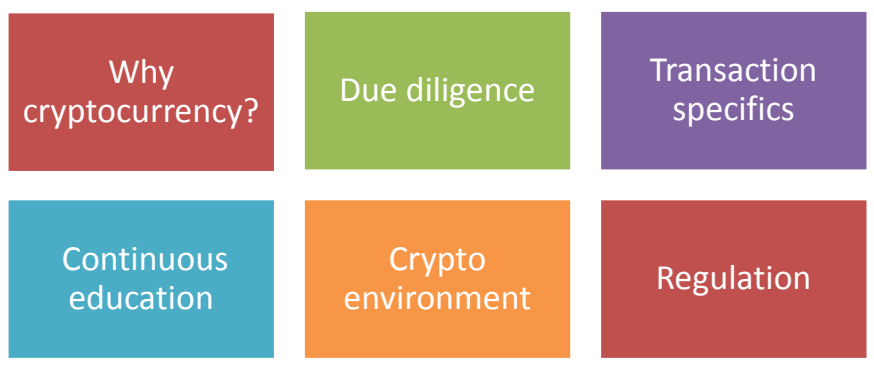

Figure 1. Questions and concepts CPAs should consider when dealing with cryptocurrency clients. 
utilize blockchain technology, but a number of different information streams will become more readily available based on this platform. Operational information, data harnessed from various devices connected to the Internet of Things, and data linked to sustainability initiatives are also prime categories where data will become more readily available. It is important to circle back to the reality that, in spite of the investment and efforts that continue to be underway in the space, complications arising from both the Internet of Things and changing regulation, including GDPR, may create as of yet unforeseen consequences [11]. This availability, however, will also present an opportunity for organizations and practitioners to engage with other technology tools and platforms as they become more present and integrated within business operations. The scope of these technology tools are not the focus of this piece but will certainly include artificial intelligence and devices connected to the Internet of Things.

Outside of the rather clear technical aspects of these new developments and platforms, accounting practitioners and professionals will have to play a larger and more forward looking role in advising clients and colleagues. Specifically, being able to offer advice and guidance to internal partners and colleagues seeking to implement emerging technologies forms a core competency that organizations across industry lines are going to require. Technology clearly does generate opportunities for organizations to be efficient and leverage data that is both generated internally and externally, but should also be balanced against what these emerging platforms are still incapable of doing. Such an analysis and understanding of technology, its opportunities, and its limitations also forms a foundation for how accounting practitioners will need to evolve to engage and confront fraud or unethical behavior in the broader cryptocurrency marketplace.

\section{The Role of Accounting in a Cryptoeconomy}

As various cryptocurrencies continue to become more widespread both in terms of investment and utilization for transactional purposes, it appears clear that the role of accounting practitioners will be transition, change, and evolve as the crypto landscape evolves. Regardless of whether practitioners are employed within public practice or private industry, several implications and ramifications of cryptocurrency implementation remain consistent. First, accounting professionals need to be well versed in how cryptocurrencies function and operate. This does not mean, however, that CPAs and other accounting professionals will be expected to become technical experts or coders. Rather, practitioners will be expected to be knowledgeable enough about cryptoassets and the cryptocurrencies to answer operational questions, advise clients on implementation issues, and most importantly, provide insights to help prevent unethical or potentially fraudulent activities.

Second, as other applications and implementations of blockchain technology, which is the underlying platform for cryptocurrencies in any sense, develop and become more widespread, accounting practitioners will also need to be aware of 
how these may drive business changes. ICOs, potentially the most high profile example of advances in blockchain technology and cryptocurrency, clearly represent a situation where unethical actors and organizations could potentially defraud investors. While the ICO market is, arguably, the most high profile example and application of advances in blockchain and cryptocurrency technology, there are other developments underway that will change how businesses operate. Decentralized autonomous organizations will, virtually without a doubt, fundamentally alter and change how organizations operate and engage with the marketplace. Governance questions, compliance with money laundering and other financial regulations, and making sure that all investors and participants in the DAO are who they represent themselves to be are issues that will need to address by quantitative professionals moving forward.

Third, and arguably the most important implication for accounting going forward is that the rise of the crypto economy will only accelerate the digitization and increasing technological integration of the profession. Whether it is the Internet of Things, artificial intelligence, cryptocurrencies, RPA tools and processes, or other automation trends, the underlying reality is that professionals are going to have to be aware of both the negatives and opportunities associated with this increasing technology integration. Additionally, and also an important part of the cryptocurrency, fraud and unethical prevention, and the broader conversation around this change is a related firm. Namely put, what changes in roles and responsibilities will be both related to the cryptocurrency movement and the accounting profession as a whole, and do these news roles indicate for practitioners.

\section{Job Opportunities and Trends Moving Forward}

An overall trend and force that should be acknowledged directly connected both to the broader cryptocurrency movement and the role that accounting practitioner will play moving forward is that practitioners will have to take full advantage and leverage technology tools. Digitization, technological integration, and the expectations of clients and customers for accounting practitioners to be well versed in these trends and tools will inevitably drive disruption and change within the profession. Included directly within this shift, however, will also be the expectation that professionals will be able to protect organizations and assets from unethical, fraudulent, or even simply error prone activities. Drilling in specifically, several opportunities and trends appear to be predictable and connected to the changing accounting landscape.

First, smart contracts and other more advanced applications connected to blockchain technology will have to be audited and examined. This does not mean that CPAs or other accounting professionals will need to understand the programming logic underpinning the smart contract itself. Rather, and circling back to the traditional role of accountants as business advisors and interpreters of information, two distinct areas appear well suited for accounting integration. The implementation of smart contracts, and ensuring that the business realities 
of the organization and business agreement are actually being translated into the smart contract coding, including making sure that no parties are exposed to undue risk or obligations. This also connects to the need for accounting professionals to assist both internal and external users to develop guidelines and reporting standards.

Second, and building off of existing aspects of general information technology controls (GITC) already familiar to accounting professionals employed both in public practice and private industry, the underlying blockchain technology and structure will also need to be examined. Again, experts should be hired to assist with the construction and development of the underlying blockchain, but blockchain technology can also provide other management professionals the key questions to ask when implementing blockchain technology. Particularly as it pertains to private blockchain technology, several core questions and pieces of information need to be assessed and answered of both the blockchain administrator and other network members. Who is the client in the context of assurance information, i.e. who is the end user who will be receiving the audit reporting and findings? A related question is which party or individual will actually receive the engagement letter?

Regardless of the specific questions or roles played by professionals within different organizations, and what oversight roles and delegated to the accounting team, the reality is that accounting practitioners will need to know how to integrate technology options internally and with client firms. While some of the technical points and perspectives will inevitably change and evolve over the passage of time, there do appear to be several points that will create future opportunities. First, auditing and analyzing control processes and procedures for accuracy, robustness, and applicability to business goals including smart contracts, ICOs, and other blockchain projects align directly with current control protocols and patterns. Second, keeping abreast of the changing regulatory landscape connected to cryptocurrency and blockchain applications will remain a core responsibility of accounting professionals for the foreseeable future. Third, and perhaps most importantly, as the uncertainty around the development and implementation of blockchain continues, accounting professionals will be sought out to advise and help prevent unethical and fraud activity across the cryptocurrency and blockchain space.

\section{Conclusion}

The rise and proliferation of cryptocurrencies throughout the accounting, finance, and other industry lines have created numerous opportunities and challenges for other management professionals as well as the accounting profession itself. Alongside the excitement and buzz that have accompanied the pace of cryptocurrency adoption, there have also been numerous instances of fraud, unethical behaviors, and errors that have cost investors billions of combined dollars. Accounting professionals, while also learning and staying informed about these emerging technology trends, also have a prime opportunity to advise 
clients and colleagues about how to prevent unethical actors from continuing to damage market actors. This does not mean that fraud or other unethical behavior will be eliminated from the marketplace, but that by remaining current and up-to-date on changes the risk of such behavior can be reduced. Cryptocurrencies have several aspects and angles that may appear confusing and open the door to liability and fraudulent, but this does not have to be the case. CPAs and other accounting professionals proactive and willing to learn, evolve, and develop in the face of a changing business landscape appear to be uniquely positioned to leverage these trends effectively to the benefit of colleagues and clients.

\section{Conflicts of Interest}

The author declares no conflicts of interest regarding the publication of this paper.

\section{References}

[1] Tysiac, K. and Drew, J. (2018) Accounting Firms: The Next Generation. Journal of Accountancy, 225, 3-9.

[2] Drew, J. (2018) Merging Accounting with "Big Data" Science. Journal of Accountancy, 226, 47-52.

[3] Sontakke, K.A. and Ghaisas, A. (2017) Cryptocurrencies: A Developing Asset Class. International Journal of Business Insights \& Transformation, 10, 10-17. https://doi.org/10.1080/1540496X.2016.1193002

[4] Jackson, O. (2018) Primer: Cryptocurrency ICO Regulation in the US. International Financial Law Review.

[5] Jackson, O. (2018) Cryptocurrency Exchanges Avoiding the US Due to Confusing Regulation. International Financial Law Review, 1.

[6] Labbé, A. (2017) PRC Regulator Blames Fraud for ICO Ban. International Financial Law Review, 1.

[7] Boeve, R. (2018) Bitcoin and Other Cryptocurrencies in Tax. International Tax Review, 1.

[8] Drew, J. (2018) Paving the Way to a New Digital World. Journal of Accountancy, 225, 32-37.

[9] Harwick, C. (2016) Cryptocurrency and the Problem of Intermediation. Independent Review, 20, 569-588.

[10] Lai, K. (2018) Blockchain as AML Tool: A Work in Progress. International Financial Law Review.

[11] Posadas Jr., D.V. (2018) The Internet of Things: The GDPR and the Blockchain May Be Incompatible. Journal of Internet Law, 21, 1-29. 